\title{
Guiding Molecularly Imprinted Polymer Design by Pharmacophore Modeling
}

\author{
Wiebke Derz ${ }^{1,2}$, Melita Fleischmann ${ }^{1,3}$ and Paul W. Elsinghorst $1,2,4, *$ (D) \\ 1 Central Institute of the Bundeswehr Medical Service Munich, 85748 Garching, Germany; \\ wiebkederz@bundeswehr.org (W.D.); melitafleischmann@bundeswehr.org (M.F.) \\ 2 Institute of Nutrition and Food Sciences, University of Bonn, 53115 Bonn, Germany \\ 3 Food Technology \& Nutrition, Management Center Innsbruck, 6020 Innsbruck, Austria \\ 4 Pharmaceutical Chemistry I, Pharmaceutical Institute, University of Bonn, 53121 Bonn, Germany \\ * Correspondence: paulelsinghorst@bundeswehr.org; Tel.: +49-89-3755849-5210
}

Citation: Derz, W.; Fleischmann, M.; Elsinghorst, P.W. Guiding Molecularly Imprinted Polymer Design by Pharmacophore Modeling. Molecules 2021, 26, 5101. https:// doi.org/10.3390/molecules26165101

Academic Editor: Giorgio Marrubini

Received: 26 July 2021

Accepted: 19 August 2021

Published: 23 August 2021

Publisher's Note: MDPI stays neutral with regard to jurisdictional claims in published maps and institutional affiliations.

Copyright: (c) 2021 by the authors. Licensee MDPI, Basel, Switzerland This article is an open access article distributed under the terms and conditions of the Creative Commons Attribution (CC BY) license (https:// creativecommons.org/licenses/by/ $4.0 /)$.

\begin{abstract}
Molecularly imprinted polymers (MIP) combine the selectivity of immunoaffinity chromatography with the robustness of common solid-phase extraction in what is referred to as molecularly imprinted solid-phase extraction (MISPE). This contribution shows how MIP design may be guided by pharmacophore modeling for the example of citrinin, which is an emerging mycotoxin from cereals. The obtained pharmacophore model allowed searching public databases for a set of citrinin-mimicking molecular surrogates. Imprinted and non-imprinted polymers were subsequently obtained through bulk and core-shell polymerization in the presence of these surrogates. Evaluation of their binding ability for citrinin and structurally related ochratoxin A revealed a promising MIP derived from rhodizonic acid. A protocol for MISPE of citrinin from cereals was subsequently developed and compared to immunoaffinity chromatography with respect to clean-up efficiency and recovery.
\end{abstract}

Keywords: mycotoxins; citrinin; core-shell polymers; molecularly imprinted polymers; MIP; MISPE; pharmacophore modeling

\section{Introduction}

Throughout the last 20 years, molecularly imprinted polymers (MIP) have gained increased interest in separation science and sample preparation [1-3]. Especially where sample preparation relies on sensitive and selective immunoaffinity chromatography (IAC), MIP appear as an attractive alternative, as they can show comparable extraction efficacy while allowing more robust handling. They do not require specific storage conditions, shelflife is in principle unrestricted, and washing with organic solvents is also possible [2,4]. As such, mycotoxin analysis is one emerging field, where IAC is gradually replaced or accompanied by molecularly imprinted solid-phase extraction (MISPE) [4]. In addition, MISPE cartridges are less cost-intensive and provide independence of commercial suppliers if produced in-house at laboratory scale. However, obtaining comparable selectivity and sensitivity in analyte binding requires the selection of a suitable surrogate template for molecular imprinting, which in the past has usually been based on visual structure comparison by individual researchers. To increase the explorable chemical space beyond this biased perspective, we suggest applying a more general approach based on pharmacophore modeling known from pharmaceutical drug discovery.

Lead identification as an early step in modern drug discovery requires the screening of large libraries of possible candidate molecules for possible ligand-receptor interaction. To reduce costs of in vitro high-throughput screening, in silico pharmacophore modeling is often applied [5]. As drug discovery programs mostly address known targets, receptorbased modeling is commonly applied, where the target receptor is constructed in silico and screened against public databases to identify possibly binding molecules. In case that no 
information on the target structure is available, but at least one ligand is known, database screening can be carried out using a three-dimensional map (pharmacophore) of possible ligand interactions.

Citrinin (CIT) is a polyketide mycotoxin produced by Aspergillus, Monascus, and Penicillium species on vegetable foodstuff, especially on rice and cereal products. Dietary supplements derived from rice fermented by Monascus purpureus for the red pigment monacolin K (lovastatin) contain considerable amounts of CIT [6]. While acute toxicity is low, long-term nephrotoxic, hepatotoxic, and cytotoxic effects depending on the frequency and duration of CIT exposure must be taken into account [7,8]. In addition, CIT often co-occurs with other mycotoxins, showing potentially harmful synergism, and a possible carcinogenic effect cannot be excluded $[9,10]$. EU legislation has set a maximum allowed limit of $2 \mathrm{mg} / \mathrm{kg}$ for dietary supplements, and the EFSA recommends a whole-diet TDI of $0.2 \mu \mathrm{g} / \mathrm{kg}$ body weight to avoid nephrotoxic effects [10,11], which is why CIT exposure should be kept as low as possible and needs to be monitored. Analysis of CIT is commonly carried out by LC-FLD and requires thorough sample clean-up usually achieved by IAC or MISPE [12-16], which both follow a similar workflow, where CIT is bound either by specific antibodies (IAC) or in microcavities using comparable molecular interactions (MISPE). Recently, CIT-selective MIP have also been applied for online MISPE as well as for the development of CIT-selective sensors [17-21]. To prepare the necessary MIP, different methods have been developed, the most simple being conventional bulk polymerization (Figure 1) [22-24].

a

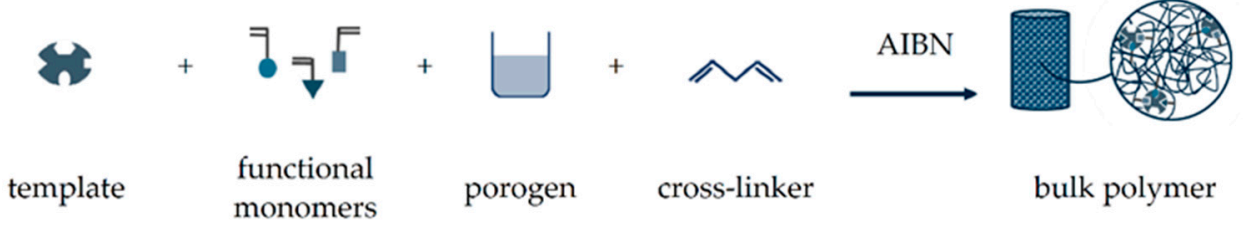

b

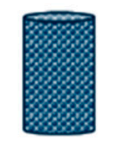

milling \& sieving

bulk polymer
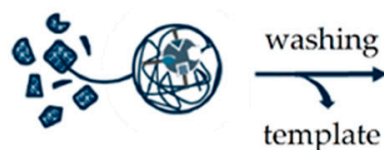

template

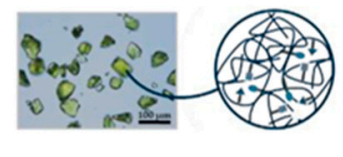

bulk MIP particles

Figure 1. Schematic illustration of MIP synthesis by bulk polymerization. First, a template, functional monomers, cross-linker, and radical starter (AIBN) are dissolved in porogen followed by heat-induced polymerization (a). The resulting polymer block is crushed, milled, and sieved to obtain uniformly sized particles, which are washed thoroughly to remove the template (b).

During polymerization, molecular imprinting itself results from a pre-polymerization complex, which is formed by non-covalent interactions between functional monomers and the selected template [25]. Understanding and optimizing the formation of this prepolymerization complex has often been the result of experimental but also of computational studies $[26,27]$. The dominating kind of interaction (polar, non-polar, or ionic) can be controlled by the organic solvent added as the porogen. Then, subsequent polymerization by cross-linking results in a solid polymer block, which is crushed, ground, and sieved to obtain particles of defined size. Final thorough washing of these particles removes the template from their microcavities to obtain the respective MIP. Another yet more elaborate way of MIP formation is by precipitation polymerization, which can provide core-shell particles by repeated application and offers several advantages including the elimination of laborious milling and sieving steps, improved template removal due to a thinner MIP layer, and more uniform polymer particles, providing better accessibility of microcavities and binding sites [28,29]. In general, the core-shell polymerization process consists of three main steps: core synthesis, core enlargement, and shell formation (Figure 2). First, 
microspherical core particles are obtained by polymerization in absence of the template, which in contrast to bulk polymerization requires a larger porogen addition to dilute the polymerization mixture $[30,31]$. This step can subsequently be repeated to enlarge the core particles before a molecularly imprinted layer is finally introduced and the template removed by washing to provide the core-shell MIP particles.

a

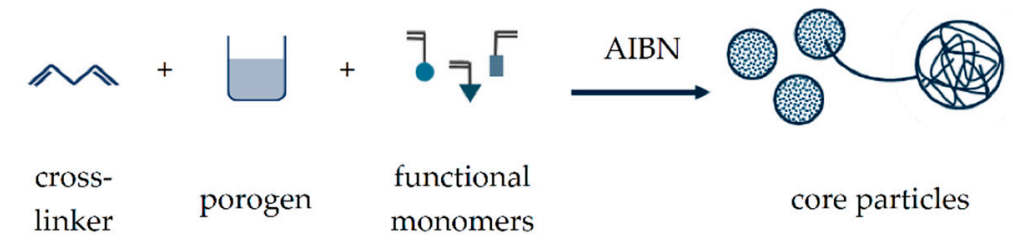

b

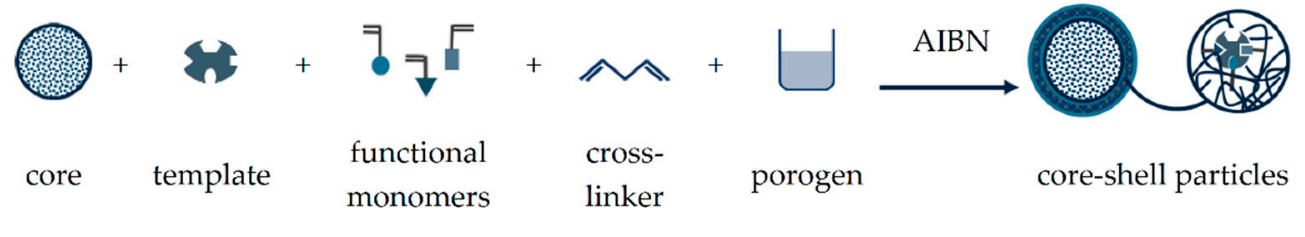

c

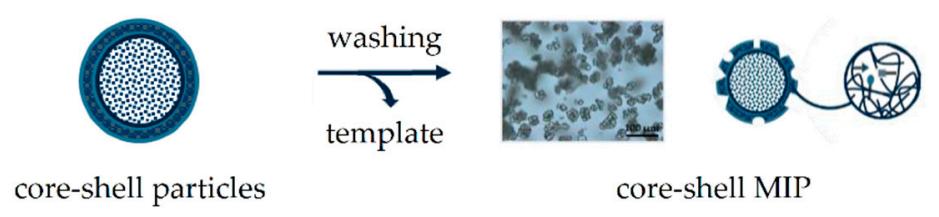

Figure 2. Schematic illustration of core-shell MIP synthesis. Core particles are obtained through heatinduced polymerization of functional monomers using a cross-linker (a), this step can be repeated to increase core size. Then, core particles are mixed with the template and functional monomers for MIP-shell formation (b), which is released by thorough washing (c).

Although molecular imprinting by the target analyte itself provides the most specific binding, carry over by polymer bleeding because of non-exhaustive washing will impair (ultra)trace analysis [15]. Instead, a surrogate template must be used, i.e., a molecule that mimics the analytes' molecular interactions and their spatial orientation, but it does not interfere with the following analysis [22]. Literature reports show that surrogate selection for CIT was predominantly driven by visual structure comparison, which is why mainly naphthoic acid derivatives have been applied [14-18]. To overcome this limited and sometimes biased perspective, computational methods such as pharmacophore modeling may offer additional insight. As depicted in Figure 3, pharmacophore modeling provides a three-dimensional map of the possible molecular interactions of the analyte using categories such as hydrogen bonding, hydrophilic, lipophilic, or ionic interactions. Then, complex matching algorithms use this interaction map (pharmacophore) to search and rank potential surrogates from public databases. The results from this search often belong to completely different compound classes that would have been missed by classic structural analogy.

As a proof of concept, we derived a pharmacophore model of citrinin in search for structurally unrelated (non-naphthoic) surrogate templates and used these for subsequent MIP and MISPE development originating from a CIT-optimized literature protocol [14]. 
<smiles>CC1=C2C(=CO[C@@H](C)[C@@H]2C)C(O)=C(C(=O)O)C1=O</smiles>

(1a)<smiles>O=c1c(O)c(O)c(=O)c(=O)c1=O</smiles>

(2a)

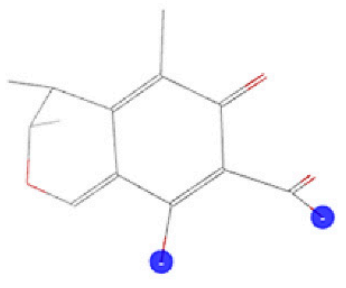

(1b)

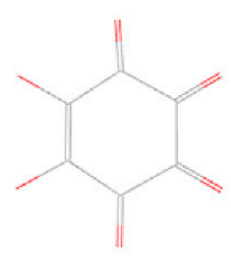

(2b)

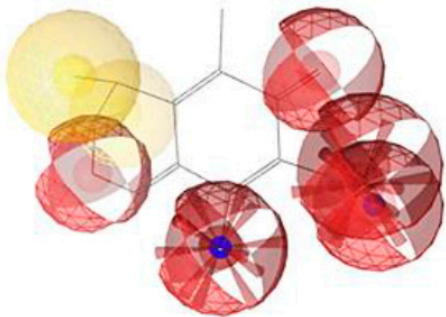

(1c)

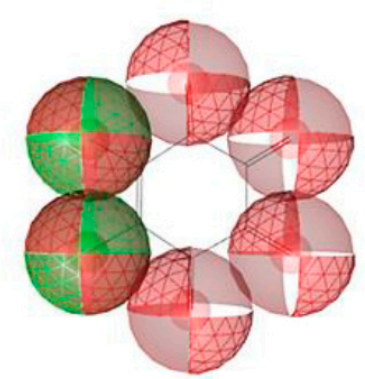

(2c)

Figure 3. Molecular structures (a), results of MMFF94 energy minimization (b), and pharmacophore models (c) of CIT (1) and the surrogate template rhodizonic acid (RHO, 2). Hydrophobic areas are marked yellow, hydrophilic ones are marked red. Areas enabling hydrogen bonding are marked green, while blue regions denote potential ionic interactions.

\section{Results and Discussion}

\subsection{Bulk Polymers}

Molecular interactions described by a pharmacophore model are subject to their chemical environment, which in the case of MIP synthesis is mainly governed by the added porogen. As such, a non-polar porogen will limit non-polar interactions while emphasizing polar interactions between surrogate templates and functional monomers and vice versa [23]. Therefore, virtual screening for potential surrogates by pharmacophore modeling must consider the appropriate porogen and be set up correctly. Considering acetonitrile, polar interactions were expected to contribute stronger to polymer binding site formation than non-polar interactions, which is why we focused our pharmacophore search on polar interactions. Using common software tools (PharmIt Search Engine [32], LigandScout [33]; see Materials and Methods for details), a list of possible surrogates was obtained, which were ranked by a match factor (pharmacophore fit score) and further filtered according to commercial availability, reasonable costs, and potential health hazards. From the top of this list, seven easy to handle, low-cost, and non-toxic surrogates were selected and applied for polymer imprinting (Table 1).

Table 1. Surrogates selected for imprinting as identified by a pharmacophore search.

\begin{tabular}{ll}
\hline Surrogate & Pharmacophore Fit Score $\mathbf{( \% )}$ \\
\hline 1,3-dihydroxy-2-naphthoic acid & 66.1 \\
rhodizonic acid dihydrate & 65.9 \\
1,4-dihydroxy-2-naphthoic acid & 65.7 \\
1-hydroxy-2-naphthoic acid & 65.6 \\
2-oxocyclohexane carboxylic acid & 56.2 \\
2-naphthoic acid & 56.0 \\
salicylic acid & 55.9 \\
\hline
\end{tabular}

MIP obtained by bulk polymerization using these surrogates were subsequently analyzed by LC-FLD for their clean-up efficiency compared to NIP (non-imprinted mock 
polymers), IAC, and common $\mathrm{C}_{18}$ SPE-columns (see Section 4 for details). Polymers imprinted by rhodizonic acid (RHO) appeared most promising with matrix recoveries around $100 \%$ comparable to IAC and superior to SPE (Table A1 (Appendix A), 94.0-127.4\% vs. 39.4-72.8\%) with notable differences between RHO-derived MIP and NIP (94.0-127.4\% vs. $21.1-126.5 \%)$. Although these initial recoveries were already satisfying, they slightly exceeded $100 \%$ in four out of five cases, indicating some remaining matrix interference. Consequently, MISPE washing was optimized to remove these matrix components effectively (see Supplementary Materials for details). Limits of detection (LOD) and quantification (LOQ) obtained after improved MISPE varied somewhat with respect to the matrix investigated except for oats, which were found to be significantly below the current maximum allowed level of $2 \mathrm{mg} / \mathrm{kg}$ for dietary supplements [11] (Table 2).

Table 2. LOD and LOQ derived for CIT quantification by MISPE using bulk MIP imprinted by RHO (following DIN 32645 [34] using a matrix-matched five point calibration, $n=3$ ).

\begin{tabular}{lll}
\hline Matrix & LOD $(\mathbf{m g} / \mathbf{k g})$ & LOQ $(\mathbf{m g} / \mathbf{k g})$ \\
\hline rice & 0.01 & 0.03 \\
rice crispies & 0.02 & 0.08 \\
wheat flour & 0.004 & 0.02 \\
pasta & 0.003 & 0.01 \\
oats & 0.1 & 0.5 \\
\hline
\end{tabular}

Recovery (55.0-96.5\%) and precision (1.9-20.4\%) data obtained at LOQ (Table 3, MISPE 1) were also acceptable and in good agreement with previous literature reports for corn (LOD/LOQ: $0.01 / 0.03 \mathrm{mg} / \mathrm{kg}$, recovery/precision: $87.1 \pm 7.2 \%$, [14]). Lower recoveries as observed in wheat products such as pasta $(55.0 \pm 13.3 \%)$ may be a result of the in situ degradation of CIT, which has been shown to decompose faster in these products [35,36]. The unexpected low recoveries obtained by IAC at LOQ may indicate superior performance of the MIP, but IAC precision was very satisfying at higher CIT concentrations (Table A1). The reusability of MISPE cartridges could be shown in the oat matrix at a CIT level of $0.5 \mathrm{mg} / \mathrm{kg}$ for at least three cycles with very good recovery $(89.3-96.5 \%)$ and precision $(0.9-1.9 \%)$, while at lower concentrations, a memory effect occurred (pasta, wheat flour, and rice products). The impact of this effect is even more significant with a CIT LOQ as low as in the pasta matrix, where MISPE cartridges cannot be reused.

Table 3. Recovery, precision, and reusability for CIT quantification by MISPE using bulk MIP imprinted by RHO (recovery as mean $\pm \mathrm{RSD}, n=6$ ).

\begin{tabular}{|c|c|c|c|c|c|}
\hline \multirow{3}{*}{ Matrix } & \multirow{3}{*}{$\begin{array}{l}\text { LOQ } \\
(\mathrm{mg} / \mathrm{kg})\end{array}$} & \multicolumn{4}{|c|}{ Recovery (\%) } \\
\hline & & \multicolumn{3}{|c|}{ MISPE } & \multirow[t]{2}{*}{ IAC } \\
\hline & & $1^{a}$ & $2^{a}$ & $3^{a}$ & \\
\hline rice & 0.03 & $62.0 \pm 4.8$ & $75.9 \pm 5.5$ & $109.0 \pm 3.6$ & - \\
\hline rice crispies & 0.08 & $88.0 \pm 3.8$ & $78.4 \pm 1.5$ & $94.7 \pm 18.2$ & $41.5 \pm 74.8$ \\
\hline wheat flour & 0.02 & $71.0 \pm 20.4$ & $65.5 \pm 7.1$ & $137.2 \pm 10.1$ & - \\
\hline pasta & 0.01 & $55.0 \pm 13.3$ & $107.2 \pm 7.1$ & $144.2 \pm 2.3$ & $57.7 \pm 29.9$ \\
\hline oats & 0.5 & $96.5 \pm 1.9$ & $96.2 \pm 0.9$ & $89.3 \pm 1.6$ & $41.6 \pm 74.7$ \\
\hline
\end{tabular}

\subsection{Core-Shell Polymers}

Although RHO-derived MIP obtained by bulk polymerization worked pretty well for CIT clean-up, grinding and washing was rather laborious and accompanied by an unavoidable loss of MIP. Therefore, we aimed to improve the manufacturing process by switching to precipitation polymerization for the production of core-shell MIP. Unfortunately, the previously used bulk polymerization mixture proved unsuitable for the production of coreshell particles, but after several attempts, we obtained RHO-imprinted core-shell particles 
suitable for MISPE using a methanol/acetone mixture as the porogen, ethylene glycol dimethacrylate (EGDMA) as the cross-linker, and $N, N$-dimethylaminoethyl methacrylate (DAEM) as the functional monomer (see Supplementary Materials for details). For comparison, also, bulk MIP and NIP comparable to these core-shell polymers were prepared using EGDMA as the cross-linker and methanol as the porogen (type II). Core-shell as well as bulk MIP and NIP were subsequently evaluated for their CIT recovery efficiency from methanol/water $(70 / 30, v / v)$ as well as from oats (Table 4$)$.

Table 4. CIT (400 ng/mL, $2 \mathrm{mg} / \mathrm{kg}$ ) recovery by MISPE using RHO-derived core-shell and type II bulk MIP and NIP (mean \pm RSD, $n=6$ ).

\begin{tabular}{llc}
\hline Matrix & Polymer & Recovery (\%) \\
\hline \multirow{3}{*}{ methanol/water $(70 / 30, v / v)$} & core-shell MIP & $97.5 \pm 2.2$ \\
& core-shell NIP & $105.5 \pm 1.8$ \\
& bulk MIP (type II) & $103.2 \pm 2.3$ \\
& bulk NIP (type II) & $103.0 \pm 1.4$ \\
\hline \multirow{4}{*}{ oats } & core-shell MIP & $97.0 \pm 2.9$ \\
& core-shell NIP & $96.9 \pm 0.3$ \\
& bulk MIP (type II) & $92.0 \pm 1.8$ \\
& bulk NIP (type II) & $90.0 \pm 1.6$ \\
\hline
\end{tabular}

Surprisingly, recovery $(90.0-105.5 \%)$ and precision $(0.3-2.9 \%)$ data were very encouraging for both type II bulk and core-shell polymers, with the LOD and LOQ of core-shell MIP and NIP resembling those previously obtained for bulk MIP. Again, recovery rates at LOQ reached very satisfying $85.1-97.9 \%$ with a precision of $1.1-3.4 \%$, regardless of imprinting (Table 5). Since imprinting by RHO is mainly driven by polar interactions, replacing aprotic acetonitrile by more polar and protic methanol may have limited the necessary interactions between the functional monomers and RHO. To confirm this hypothesis, core-shell MIPs and NIPs were also prepared using acetonitrile as the porogen. The resulting particles were much finer than the ones obtained with methanol, and the polymer was very voluminous with a fluffy texture. Subsequent MISPE revealed an $89.2 \pm 1.3 \%$ to $74.5 \pm 2.8 \%$ difference between these MIP and NIP, supporting the hypothesis of imprinting suppression by porogen switching from acetonitrile to methanol.

Table 5. LOD and LOQ derived for CIT quantification by MISPE using core-shell MIP imprinted by $\mathrm{RHO}$ (matrix-matched five-point calibration following DIN 32645, recovery as mean $\pm \mathrm{RSD}, n=3$ ).

\begin{tabular}{lllll}
\hline Matrix & Type & LOD $(\mathbf{m g} / \mathbf{k g})$ & LOQ $(\mathbf{m g} / \mathbf{k g})$ & Recovery $(\%)$ \\
\hline \multirow{2}{*}{ pasta } & MIP & 0.014 & 0.048 & $96.0 \pm 1.3$ \\
& NIP & 0.011 & 0.037 & $97.9 \pm 3.4$ \\
\hline \multirow{2}{*}{ oats } & MIP & 0.12 & 0.45 & $85.1 \pm 1.8$ \\
& NIP & 0.15 & 0.55 & $97.6 \pm 1.1$ \\
\hline
\end{tabular}

\subsection{Clean-Up of Ochratoxin A}

The structural similarity to CIT suggested that the prepared core-shell polymers might also be able to bind ochratoxin A (OTA). Notably, MISPE of OTA using methanol/water $(70 / 30, v / v)$ as well as oat extracts revealed very acceptable recovery and precision data with slightly better performance of NIP (Table 6). Recoveries obtained in combination experiments (CIT: $89.3 \pm 4.0 \%$, OTA: $89.0 \pm 2.7 \%$ ) were very satisfying and demonstrate the applicability of these core-shell NIPs for the simultaneous clean-up of these two mycotoxins CIT and OTA (Figure 4). 
Table 6. Recovery and precision of OTA ( $30 \mathrm{ng} / \mathrm{mL})$ quantification by MISPE using core-shell MIP and NIP imprinted by RHO (mean \pm RSD, $n=6$ ).

\begin{tabular}{lll}
\hline Matrix & Type & Recovery (\%) \\
\hline \multirow{2}{*}{ methanol/water $(70 / 30, v / v)$} & MIP & $82.7 \pm 5.0$ \\
& NIP & $95.8 \pm 7.3$ \\
\hline \multirow{2}{*}{ oats } & MIP & $76.8 \pm 3.9$ \\
& NIP & $88.6 \pm 1.2$ \\
\hline
\end{tabular}

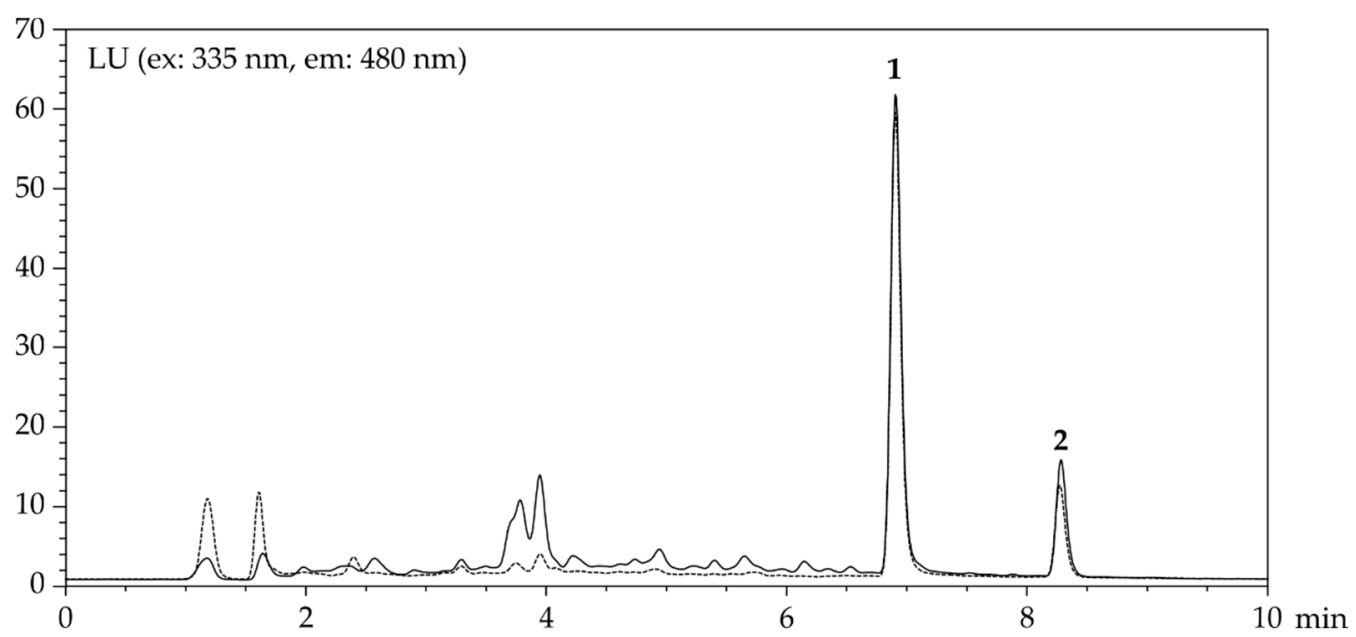

Figure 4. Chromatograms of CIT $(\mathbf{1}, 400 \mathrm{ng} / \mathrm{mL})$ and OTA $(\mathbf{2}, 30 \mathrm{ng} / \mathrm{mL})$ of oats spiked before (一) and after (---) MISPE (see Materials and Methods for details).

\section{Conclusions}

The identification of suitable surrogate templates plays a central role in MIP design for trace analysis. While MIP design strategies optimizing the pre-polymerization complex (monomer mixture composition) have been aided by computational simulations before [27,37], pharmacophore modeling goes one step ahead, mapping the analytes' interaction potential to discover possible surrogates from chemical space that would have been missed by traditional methods. Even when starting from an already optimized MIP production process, a pharmacophore-based surrogate search can be applied to follow-up improvement studies. Subsequent in-house MIP production offers the selectivity usually associated with IAC at the affordable price of common SPE with independence from commercial suppliers or specific storage conditions. The bulk polymers reported here for CIT clean-up are shelf-stable while offering robust handling. When switching from bulk to core-shell MIP production, careful attention must be paid to a possible change in porogen. Changing polarities or protic/aprotic conditions will affect the pharmacophore search, as molecular interactions can be susceptible to even slight modifications. By chance, an additional NIP was discovered that is capable of simultaneously recovering CIT and OTA from oats and common extract solvents.

\section{Materials and Methods}

\subsection{Reagents and Materials}

Citrinin (CIT), 1-hydroxy-2-naphthoic acid, 1,4-dihydroxy-2-naphthoic acid, 2,2-azobis isobutyronitrile (AIBN), trimethylolpropane trimethacrylate (TRIM), methacrylic acid (MAA), and PBS tablets were obtained from Sigma (Steinheim, Germany). Barium chloride, $\mathrm{N}, \mathrm{N}$-dimethylaminoethyl methacrylate (DAEM), ethylene glycol dimethacrylate (EGDMA), methacrylamide (MAM), salicylic acid, acetic acid, ammonia ( $25 \%$ aq.), sodium hydroxide solution (1M), orthophosphoric acid, and Tween 20 were purchased from Merck (Darmstadt, Germany). LC-grade acetonitrile and methanol were from VWR (Langenfeld, Germany), 
acetone was from Honeywell (Seelze, Germany). 2-Naphthoic acid was obtained from Acros Organics (Geel, Belgium), 2-oxocyclohexane carboxylic acid was obtained from Fluorochem (Karlsruhe, Germany), and rhodizonic acid dehydrate (RHO) was obtained from abcr (Karlsruhe, Germany). Ochratoxin A (OTA) was provided by HPC Standards (Borsdorf, Germany) and EASI-EXTRACT ${ }^{\circledR}$ CITRININ columns were provided by RBiopharm Rhone Ltd. (Glasgow, UK). Cereal products (wheat flour, pasta, rice, whole grain rice crispies, and oat flakes) were purchased from local markets and did not contain detectable levels of CIT. CIT stock and working solution were prepared according to DIN EN 17203 [38] and stored in acid-washed amber vials at $-20^{\circ} \mathrm{C}$.

\subsection{Pharmacophore Modeling}

Briefly, a three-dimensional structure file (SDF) of CIT was downloaded from PubChem (CID: 54680783) and submitted to the PharmIt Search Engine [32] to generate a corresponding pharmacophore model. PharmIt independently identifies possible interactions and classifies them as hydrophobic or polar/ionic (further features can be added manually if necessary). In case of CIT, five hydrogen acceptors, one hydrogen donor, which is at the same time an anionic interaction site, and four hydrophobic areas were detected. Considering the applied porogen, formation of the pre-polymerization complex was expected to be mainly driven by polar and ionic interactions. Hydrophobic interactions were consequently considered irrelevant and excluded manually before a pharmacophore search was carried out accessing public databases (PubChem, ZINCPharmer) for possible surrogates [39]. Results from this screening were subsequently downloaded and joined into a local database using LigandScout [33], which provides MMFF94 energy minimization of input molecules generating reasonable conformers of each hit. By comparing this local database with an MMFF94 energy-minimized pharmacophore model of CIT, results were ranked according to the pharmacophore fit score offered by LigandScout. Best matching, commercially available pharmacophores were subsequently used for polymer imprinting.

\subsection{Bulk Polymer Synthesis}

MIP were prepared by thermoinitiated bulk polymerization according to Appell et al. [14]. Each batch consisted of two headspace vials $(20 \mathrm{~mL})$ that were charged with RHO $(200 \mathrm{mg}$, $1 \mathrm{mmol}$ ) or, when comparing previously identified surrogates, the respective surrogate template $(1 \mathrm{mmol})$, acetone $(1.875 \mathrm{~mL})$, acetonitrile $(5.625 \mathrm{~mL})$, and DAEM $(0.675 \mathrm{~mL}$, $4 \mathrm{mmol}$ ). The vials were vortexed, sealed, and sonicated for $15 \mathrm{~min}$ before storage at $4{ }^{\circ} \mathrm{C}$ for $4 \mathrm{~h}$. Next, TRIM ( $\left.4.79 \mathrm{~mL}, 15 \mathrm{mmol}\right)$ was added, and the mixture was flushed with nitrogen for $5 \mathrm{~min}$ followed by AIBN ( $300 \mathrm{mg}, 1.8 \mathrm{mmol}$ ). The vials were sealed and vortexed again before polymerization was allowed to proceed for $48 \mathrm{~h}$ in a water bath at $55^{\circ} \mathrm{C}$. The resulting bulk polymers were released as monoliths by careful crushing of the glass vials, after which they were finely ground and sieved to achieve particles less than $77 \mu \mathrm{m}$ in diameter. To prevent static charging, a few drops of methanol were added while grinding. Particles were subsequently suspended in a mixture of methanol $(50 \mathrm{~mL})$ and water $(100 \mathrm{~mL})$; then, they were subjected to centrifugation $\left(150 \times g, 20^{\circ} \mathrm{C}\right.$, $5 \mathrm{~min}$ ) using polypropylene tubes, and the supernatant was discarded. Washing with water $(6 \times 150 \mathrm{~mL})$ followed by acetone $(1 \times 25 \mathrm{~mL})$ was repeated a total of seven times. Superfine particles were finally removed by suspension in acetone $(6 \times 50 \mathrm{~mL})$, which was allowed to settle for 2 min every time. MIP and NIP (non-imprinted mock polymers) were dried by vacuum filtration. The successful release of RHO during washing was monitored by visual inspection and by precipitation with aqueous barium chloride $(0.25 \mathrm{~g} / \mathrm{mL}$; a reddish precipitate insoluble in hydrochloric acid $(0.1 \mathrm{M})$ is obtained when a drop of washing water is placed on a filter paper followed by a drop of the barium chloride solution [40]). For routine MIP production, visual monitoring of the wash-out process was considered sufficient, as the possibly remaining RHO does not interfere with CIT determination. However, precipitate formation provides additional proof that $\mathrm{RHO}$ deterioration as a result of the applied reaction conditions can be excluded. 


\subsection{Core-Shell Polymer Synthesis}

The preparation of core-shell polymers followed previously published polymerization protocols [29,31,41-43]. Briefly, synthesis was carried out in three steps: core synthesis, core enlargement, and shell formation. To form the core first, EGDMA $(1.895 \mathrm{~mL}, 10 \mathrm{mmol})$ and DAEM $(0.507 \mathrm{~mL}, 3 \mathrm{mmol})$ were dissolved in a mixture of acetone $(3.75 \mathrm{~mL})$ and methanol $(11.25 \mathrm{~mL})$ placed in a headspace vial $(50 \mathrm{~mL})$. Following sonication for $15 \mathrm{~min}$, the mixture was cooled to $0{ }^{\circ} \mathrm{C}$ for $1 \mathrm{~h}$, AIBN $(164 \mathrm{mg}, 1 \mathrm{mmol})$ was added, and the mixture was flushed with nitrogen for $10 \mathrm{~min}$. Subsequent polymerization was carried out at $55^{\circ} \mathrm{C}$ for $5 \mathrm{~h}$ using a water bath while stirring at $500 \mathrm{rpm}$. Following centrifugation $(133 \times g$, $20^{\circ} \mathrm{C}, 1 \mathrm{~min}$ ) and discarding of the supernatant, core enlargement was carried out using a freshly prepared porogen/cross-linker/monomer/initiator mixture added to the core particles. Final shell formation was achieved using DAEM $(1.014 \mathrm{~mL}, 6 \mathrm{mmol})$ and RHO (412 mg, $2 \mathrm{mmol})$, which were premixed in a separate vial containing acetone $(6.25 \mathrm{~mL})$ and methanol $(18.75 \mathrm{~mL})$, sonicated for $15 \mathrm{~min}$, and cooled down to $0{ }^{\circ} \mathrm{C}$ for $1 \mathrm{~h}$. EGDMA $(1.895 \mathrm{~mL}, 10 \mathrm{mmol})$ was added, and the whole mixture was transferred to the headspace vial containing the previously prepared polymer cores obtained by centrifugation $(133 \times g$, $\left.20{ }^{\circ} \mathrm{C}, 1 \mathrm{~min}\right)$. After sonication for $5 \mathrm{~min}$, AIBN ( $\left.164 \mathrm{mg}, 1 \mathrm{mmol}\right)$ was added, and the vial was flushed with nitrogen for $10 \mathrm{~min}$ followed by polymerization as described above. The resulting core-shell particles were washed by suspension/centrifugation $\left(133 \times g, 20^{\circ} \mathrm{C}\right.$, $5 \mathrm{~min}$ ) using polypropylene tubes as shown in Table 7 and finally dried by vacuum filtration (see Supplementary Materials for further details).

Table 7. Washing steps for core-shell MIP and NIP production.

\begin{tabular}{lllll}
\hline $\mathbf{1 .}$ & $\mathbf{2 .}$ & $\mathbf{3 .}$ & $\mathbf{4 .}$ & $\mathbf{5 .}$ \\
\hline methanol & water & methanol & water & acetone \\
$1 \times 50 \mathrm{~mL}$ & $5 \times 50 \mathrm{~mL}$ & $5 \times 50 \mathrm{~mL}$ & $10 \times 50 \mathrm{~mL}$ & $1 \times 50 \mathrm{~mL}$ \\
\hline
\end{tabular}

\subsection{Cartridge Packing}

Bulk or core-shell polymers were packed for MISPE using the slurry method. Polymer particles (150 $\pm 1 \mathrm{mg})$ were placed into an empty polypropylene SPE cartridge equipped with a bottom frit and suspended in $2 \mathrm{~mL}$ of methanol. The solvent was removed by vacuum filtration, and a second frit was stacked onto the swollen polymer. Cartridges were either directly used for MISPE or dried and stored at room temperature protected from light (polymer particles will shrink to their original size but will recover when moistened again).

\subsection{Extraction and MISPE}

Cereal products $(40 \mathrm{~g})$ were finely ground, suspended in methanol/water $(70 / 30, v / v$, $200 \mathrm{~mL}$ ), and shaken horizontally for $1 \mathrm{~h}$ at $200 \mathrm{rpm}$ using polypropylene tubes. Centrifugation $\left(3774 \times g, 20^{\circ} \mathrm{C}, 10 \mathrm{~min}\right)$ followed by microfiltration (PTFE, $\left.0.45 \mu \mathrm{m}\right)$ provided a supernatant suitable for immediate analysis. If required, spiking with CIT $(400 \mathrm{ng} / \mathrm{mL})$ or OTA $(30 \mathrm{ng} / \mathrm{mL}$ ) was carried out afterwards to exclude any influence by extraction recovery. MISPE cartridges were flushed with a mixture of $2 \%$ ammonia ( $25 \%$ aq.) in methanol ( $3 \mathrm{~mL}$ ) followed by conditioning with methanol/water $(70 / 30, v / v, 3 \mathrm{~mL})$. Samples $(1 \mathrm{~mL})$ were subsequently loaded onto the columns followed by washing with $1 \%$ acetic acid in methanol $(0.8 \mathrm{~mL})$. CIT and OTA were eluted with $2 \%(25 \%$ aq.) ammonia in methanol ( $2 \mathrm{~mL}, 2 \times 0.8 \mathrm{~mL}$ with backflushing for core-shell polymers) at a flow rate of $2 \mathrm{~mL} / \mathrm{min}$ (approximately 1 drop $/ 2 \mathrm{~s}$ ), collected in amber vials and evaporated to dryness using a vacuum concentrator (RVC 2-18, Christ, Osterode, Germany) at $50{ }^{\circ} \mathrm{C}\left(40^{\circ} \mathrm{C}\right.$ for core-shell polymers). Extracts were taken up in $10 \mathrm{mM}$ orthophosphoric acid/acetonitrile $(80 / 20, v / v$, $1 \mathrm{~mL}$ ) or methanol/water (50/50, $v / v, 1 \mathrm{~mL}$; core-shell polymers), filtered (PTFE, $0.45 \mu \mathrm{m}$ ), and subjected to LC analysis $(20 \mu \mathrm{L})$. 


\subsection{Immunoaffinity Chromatography}

IAC was carried out following the supplier's instructions [13]. Briefly, samples (2 mL) were diluted with PBS to a final volume of $20 \mathrm{~mL}$, of which $10 \mathrm{~mL}$ were loaded onto the IAC columns at a flow rate of approximately $2 \mathrm{~mL} / \mathrm{min}$. The columns were washed with $0.1 \%$ Tween 20 (10 $\mathrm{mL}$ in $10 \mathrm{mM}$ orthophosphoric acid) followed by $10 \mathrm{mM}$ orthophosphoric acid $(10 \mathrm{~mL}, \mathrm{pH} 2.5)$. CIT was eluted using methanol $(1 \mathrm{~mL})$ followed by water $(1 \mathrm{~mL})$ into amber vials and subjected to LC analysis $(40 \mu \mathrm{L})$.

\subsection{Quantification of Citrinin and Ochratoxin A}

Liquid chromatography of CIT and OTA was carried out using an Agilent (Waldbronn, Germany) $1200 \mathrm{LC}$ system equipped with a fluorescence detector $\left(\lambda_{\mathrm{ex}}=335 \mathrm{~nm}\right.$, $\left.\lambda_{\mathrm{em}}=480 \mathrm{~nm}\right)$. Chromatographic separation was achieved on a Phenomenex Luna $\mathrm{C}_{18}$ column (4.6 $\times 100 \mathrm{~mm}, 3 \mu \mathrm{m}$; Aschaffenburg, Germany) using gradient elution (A: $10 \mathrm{mM}$ orthophosphoric acid, B: acetonitrile; $\mathrm{min} / \% \mathrm{~B}: 0 / 20,6 / 60,9 / 30,12 / 20)$ at $40{ }^{\circ} \mathrm{C}$ and a flow rate of $1 \mathrm{~mL} / \mathrm{min}$. Following DIN 32645 [34], limits of detection (LOD) and quantification (LOQ) were determined using three-fold matrix-matched, equidistant five-point calibration curves within the expected LOQ range (Table 8). Recovery and precision data for each matrix were subsequently obtained at the determined LOQ $(n=6)$.

Table 8. Matrix-matched calibration levels for bulk and core-shell MIP and NIP.

\begin{tabular}{lll}
\hline Polymer Type & Matrix & Calibration Range $(\mathrm{mg} / \mathbf{k g})$ \\
\hline & rice & $0.025,0.050,0.075,0.100,0.125$ \\
bulk MIP & rice crispies & $0.05,0.10,0.15,0.20,0.25$ \\
& wheat flour & $0.025,0.050,0.075,0.100,0.125$ \\
& pasta & $0.025,0.050,0.075,0.100,0.125$ \\
oats & $0.5,1.0,1.5,2.0,2.5$ \\
\hline \multirow{2}{*}{ core-shell MIP/NIP } & pasta & $0.025,0.050,0.075,0.100,0.125$ \\
& oats & $0.5,1.0,1.5,2.0,2.5$ \\
\hline
\end{tabular}

Supplementary Materials: The following are available online. Figure S1: CIT short-term stability. Figure S2: CIT and OTA long-term stability. Table S1: Optimization of MISPE elution using bulk polymers imprinted by 1,4-dihydroxy-2-naphthoic acid. Table S2: Optimization of MISPE washing as estimated by recovery and FLD noise. Table S3: Optimization of washing volume. Table S4: Different combinations of porogen, cross-linker, and functional monomers and their effect on the size, form, and behavior of core-shell MIP imprinted by RHO.

Author Contributions: Conceptualization, W.D., M.F. and P.W.E.; methodology, W.D.; validation, W.D. and M.F.; investigation, W.D. and M.F.; resources, P.W.E.; writing—original draft preparation, W.D; writing-review and editing, P.W.E.; visualization, W.D. and P.W.E.; supervision, P.W.E.; project administration, P.W.E. All authors have read and agreed to the published version of the manuscript.

Funding: This research received no external funding.

Institutional Review Board Statement: Not applicable.

Informed Consent Statement: Not applicable.

Data Availability Statement: The data presented in this study are available in Supplementary Materials.

Acknowledgments: The technical support by Daniel Mesfun and Jan Lang is gratefully acknowledged.

Conflicts of Interest: The authors declare no conflict of interest.

Sample Availability: Samples of the MIP are available from the authors. 


\section{Appendix A}

Table A1. CIT ( $400 \mathrm{ng} / \mathrm{mL}, 2 \mathrm{mg} / \mathrm{kg}$ ) recoveries achieved by clean-up of rice, rice crispies, wheat flour, pasta, and oats applying MISPE, IAC, and SPE. Polymers for MISPE were imprinted using surrogates identified by pharmacophore modeling (mean \pm RSD, $n=6$ ).

\begin{tabular}{|c|c|c|c|c|c|c|}
\hline \multirow{2}{*}{ Clean-Up } & \multirow{2}{*}{ Imprinting Surrogate } & \multicolumn{5}{|c|}{ Recovery (\%) } \\
\hline & & Rice & Rice Crispies & Wheat Flour & Pasta & Oats \\
\hline \multirow{8}{*}{ MISPE } & 2-naphthoic acid & $49.5 \pm 6.6$ & $71.1 \pm 53.6$ & $32.0 \pm 2.2$ & $66.4 \pm 13.9$ & $90.7 \pm 2.1$ \\
\hline & 1-hydroxy-2-naphthoic acid & $60.8 \pm 15.9$ & $119.1 \pm 6.0$ & $62.4 \pm 20.9$ & $72.8 \pm 4.3$ & $76.4 \pm 5.0$ \\
\hline & 1,3-dihydroxy-2-naphthoic acid & $72.9 \pm 4.4$ & $59.2 \pm 12.2$ & $38.3 \pm 15.0$ & $99.8 \pm 6.1$ & $79.8 \pm 2.5$ \\
\hline & 1,4-dihydroxy-2-naphthoic acid & $88.0 \pm 1.7$ & $88.7 \pm 34.8$ & $67.8 \pm 17.2$ & $125.9 \pm 0.3$ & $94.0 \pm 3.8$ \\
\hline & salicylic acid & $54.5 \pm 4.4$ & $120.8 \pm 3.4$ & $36.5 \pm 13.3$ & $120.8 \pm 1.3$ & $87.8 \pm 0.9$ \\
\hline & 2-oxocyclohexane carboxylic acid & $51.6 \pm 12.0$ & $116.6 \pm 2.7$ & $30.2 \pm 10.6$ & $114.8 \pm 2.9$ & $77.2 \pm 5.8$ \\
\hline & rhodizonic acid dihydrate & $94.0 \pm 1.2$ & $118.4 \pm 4.0$ & $111.7 \pm 2.6$ & $127.4 \pm 2.6$ & $114.0 \pm 0.8$ \\
\hline & non-imprinted & $61.4 \pm 6.5$ & $126.5 \pm 1.1$ & $21.1 \pm 18.8$ & $91.5 \pm 24.4$ & $78.6 \pm 3.4$ \\
\hline IAC & - & $97.9 \pm 9.4$ & $121.4 \pm 2.4$ & $87.1 \pm 2.3$ & $114.3 \pm 10.5$ & $96.5 \pm 8.9$ \\
\hline $\operatorname{SPE}\left(C_{18}\right)$ & - & $50.9 \pm 4.6$ & $72.8 \pm 17.9$ & $45.3 \pm 7.5$ & $50.5 \pm 13.8$ & $39.4 \pm 22.7$ \\
\hline
\end{tabular}

\section{References}

1. BelBruno, J.J. Molecularly Imprinted Polymers. Chem. Rev. 2019, 119, 94-119. [CrossRef] [PubMed]

2. Turiel, E.; Martín-Esteban, A. Molecularly Imprinted Polymers for Sample Preparation: A Review. Anal. Chim. Acta 2010, 668, 87-99. [CrossRef] [PubMed]

3. Gao, M.; Gao, Y.; Chen, G.; Huang, X.; Xu, X.; Lv, J.; Wang, J.; Xu, D.; Liu, G. Recent Advances and Future Trends in the Detection of Contaminants by Molecularly Imprinted Polymers in Food Samples. Front. Chem. 2020, 8, 616326. [CrossRef] [PubMed]

4. Kralj Cigić, I.; Prosen, H. An Overview of Conventional and Emerging Analytical Methods for the Determination of Mycotoxins. Int. J. Mol. Sci. 2009, 10, 62-115. [CrossRef]

5. Yang, S.-Y. Pharmacophore Modeling and Applications in Drug Discovery: Challenges and Recent Advances. Drug Discov. Today 2010, 15, 444-450. [CrossRef]

6. López Sáncheza, P.; de Nijsa, M.; Spanjerb, M.; Pietric, A.; Bertuzzic, T.; Starski, A.; Postupolski, J.; Castellari, M.; Hortós, M. Generation of Occurrence Data on Citrinin in Food. EFSA Support. Publ. 2017, 14, 1177E. [CrossRef]

7. Ambrose, A.M.; Deeds, F. Some Toxicological and Pharmacological Properties of Citrinin. J. Pharmacol. Exp. Ther. 1946, 88, 173-186.

8. Gayathri, L.; Karthikeyan, B.S.; Rajalakshmi, M.; Dhanasekaran, D.; Li, A.P.; Akbarsha, M.A. Metabolism-Dependent Cytotoxicity of Citrinin and Ochratoxin A Alone and in Combination as Assessed Adopting Integrated Discrete Multiple Organ Co-Culture (IdMOC). Toxicol. In Vitro 2018, 46, 166-177. [CrossRef]

9. Ostry, V.; Malir, F.; Ruprich, J. Producers and Important Dietary Sources of Ochratoxin A and Citrinin. Toxins 2013, 5, 1574-1586. [CrossRef]

10. EFSA. Scientific Opinion on the Risks for Public and Animal Health Related to the Presence of Citrinin in Food and Feed. EFSA J. 2012, 10, 2605. [CrossRef]

11. European Commission. Commission Regulation (EU) No. 212/2014 Amending Regulation (EC) No. 1881/2006 as Regards Maximum Levels of the Contaminant Citrinin in Food Supplements Based on Rice Fermented with Red Yeast Monascus purpureus. 2014. Available online: https:/ / eur-lex.europa.eu/eli/reg/2014/212/oj (accessed on 25 July 2021).

12. Atapattu, S.N.; Poole, C.F. Recent Advances in Analytical Methods for the Determination of Citrinin in Food Matrices. J. Chromatogr. A 2020, 1627, 461399. [CrossRef] [PubMed]

13. Marley, E.; Brown, P.; Leeman, D.; Donnelly, C. Analysis of Citrinin in Cereals, Red Yeast Rice Dietary Supplement, and Animal Feed by Immunoaffinity Column Cleanup and LC with Fluorescence Detection. J. AOAC Int. 2016, 99, 1025-1031. [CrossRef] [PubMed]

14. Appell, M.; Jackson, M.A.; Wang, L.C.; Bosma, W.B. Determination of Citrinin Using Molecularly Imprinted Solid Phase Extraction Purification, HPLC Separation, and Fluorescence Detection. J. Liq. Chromatogr. Relat. Technol. 2015, 38, 1815-1819. [CrossRef]

15. Guo, B.-Y.; Wang, S.; Ren, B.; Li, X.; Qin, F.; Li, J. Citrinin Selective Molecularly Imprinted Polymers for SPE. J. Sep. Sci. 2010, 33, 1156-1160. [CrossRef] [PubMed]

16. Urraca, J.L.; Huertas-Pérez, J.F.; Cazorla, G.A.; Gracia-Mora, J.; García-Campaña, A.M.; Moreno-Bondi, M.C. Development of Magnetic Molecularly Imprinted Polymers for Selective Extraction: Determination of Citrinin in Rice Samples by Liquid Chromatography with UV Diode Array Detection. Anal. Bioanal. Chem. 2016, 408, 3033-3042. [CrossRef] [PubMed]

17. Lhotská, I.; Kholová, A.; Machyňáková, A.; Hroboňová, K.; Solich, P.; Švec, F.; Šatínský, D. Preparation of Citrinin-Selective Molecularly Imprinted Polymer and Its Use for on-Line Solid-Phase Extraction Coupled to Liquid Chromatography. Anal. Bioanal. Chem. 2019, 411, 2395-2404. [CrossRef] 
18. Fang, G.; Liu, G.; Yang, Y.; Wang, S. Quartz Crystal Microbalance Sensor Based on Molecularly Imprinted Polymer Membrane and Three-Dimensional Au Nanoparticles@mesoporous Carbon CMK-3 Functional Composite for Ultrasensitive and Specific Determination of Citrinin. Sens. Actuators B Chem. 2016, 230, 272-280. [CrossRef]

19. Sobiech, M.; Luliński, P.; Wieczorek, P.P.; Marć, M. Quantum and Carbon Dots Conjugated Molecularly Imprinted Polymers as Advanced Nanomaterials for Selective Recognition of Analytes in Environmental, Food and Biomedical Applications. Trends Anal. Chem. 2021, 142, 116306. [CrossRef]

20. Akyıldırım, O.; Kardaş, F.; Beytur, M.; Yüksek, H.; Atar, N.; Yola, M.L. Palladium Nanoparticles Functionalized Graphene Quantum Dots with Molecularly Imprinted Polymer for Electrochemical Analysis of Citrinin. J. Mol. Liq. 2017, 243 , 677-681. [CrossRef]

21. Hu, X.; Liu, Y.; Xia, Y.; Zhao, F.; Zeng, B. A Novel Ratiometric Electrochemical Sensor for the Selective Detection of Citrinin Based on Molecularly Imprinted Poly(Thionine) on Ionic Liquid Decorated Boron and Nitrogen Co-Doped Hierarchical Porous Carbon. Food Chem. 2021, 363, 130385. [CrossRef] [PubMed]

22. Qiao, F.; Sun, H.; Yan, H.; Row, K.H. Molecularly Imprinted Polymers for Solid Phase Extraction. Chromatographia 2006, 64, 625-634. [CrossRef]

23. Vasapollo, G.; Sole, R.D.; Mergola, L.; Lazzoi, M.R.; Scardino, A.; Scorrano, S.; Mele, G. Molecularly Imprinted Polymers. Int. J. Mol. Sci. 2011, 12, 5908-5945. [CrossRef]

24. Whitcombe, M.J.; Kirsch, N.; Nicholls, I.A. Molecular Imprinting Science and Technology: A Survey of the Literature for the Years 2004-2011. J. Mol. Recognit. 2014, 27, 297-401. [CrossRef]

25. Janczura, M.; Luliński, P.; Sobiech, M. Imprinting Technology for Effective Sorbent Fabrication: Current State-of-Art and Future Prospects. Materials 2021, 14, 1850. [CrossRef] [PubMed]

26. Golker, K.; Olsson, G.D.; Nicholls, I.A. The Influence of a Methyl Substituent on Molecularly Imprinted Polymer Morphology and Recognition-Acrylic Acid versus Methacrylic Acid. Eur. Polym. J. 2017, 92, 137-149. [CrossRef]

27. Janczura, M.; Sobiech, M.; Giebułtowicz, J.; Luliński, P. Computational and Experimental Designing of Imprinted Sorbent for the Determination of Nitroxidative Stress Products: An Analysis of 4-Hydroxyphenylacetic Acid Conversion. J. Mater. Sci. 2021, 56, 8439-8460. [CrossRef]

28. Semong, O.; Batlokwa, B.S. Development of an Aflatoxin B1 Specific Molecularly Imprinted Solid Phase Extraction Sorbent for the Selective Pre-Concentration of Toxic Aflatoxin B1 from Child Weaning Food, Tsabana. Mol. Impr. 2017, 5, 1-15. [CrossRef]

29. Ye, L. Molecular Imprinting: Principles and Applications of Micro- and Nanostructured Polymers; Pan Stanford Publications: Singapore, 2013.

30. Ramli, R.A.; Laftah, W.A.; Hashim, S. Core-Shell Polymers: A Review. RSC Adv. 2013, 3, 15543-15565. [CrossRef]

31. Renkecz, T.; Horvarth, V.; Horvai, G. Molecularly imprinted polymers for chromatography and related techniques. In Handbook of Molecularly Imprinted Polymers; Alvarez-Lorenzo, C., Concheiro, A., Eds.; Smithers Rapra Technology: Shawbury, UK, 2013; pp. 141-196.

32. Sunseri, J.; Koes, D.R. Pharmit: Interactive exploration of chemical space. Nucleic Acids Res. 2016, 44, W442-W448. [CrossRef] [PubMed]

33. Wolber, G.; Langer, T. LigandScout: 3-D pharmacophores derived from protein-bound ligands and their use as virtual screening filters. J. Chem. Inf. Model. 2005, 45, 160-169. [CrossRef]

34. DIN 32645: Decision Limit, Detection Limit and Determination Limit under Repeatability Conditions: Terms, Methods, Evaluation. 2008. Available online: https:/ /www.beuth.de/en/standard/din-32645/110729574 (accessed on 25 July 2021). [CrossRef]

35. Wilson, D.M. Citrinin: Analysis and occurrence. In Mycotoxins, Wood Decay, Plant Stress, Biocorrosion, and General Biodeterioration; Llewellyn, G.C., Dashek, W.V., O’Rear, C.E., Eds.; Springer: Boston, MA, USA, 1994; pp. 65-73.

36. Schneider, C. Vorkommen und Nachweis von Citrinin in Nahrungsmitteln Pflanzlicher Herkunft. Ph.D. Thesis, Ludwig Maximilian University, Munich, Germany, 2007.

37. Cowen, T.; Busato, M.; Karim, K.; Piletsky, S.A. In Silico Synthesis of Synthetic Receptors: A Polymerization Algorithm. Macromol. Rapid Commun. 2016, 37, 2011-2016. [CrossRef]

38. DIN EN 17203: Determination of Citrinin in Food by Liquid Chromatography Tandem Mass Spectrometry (LC-MS/MS). 2021. Available online: https:/ / www.beuth.de/en/standard/din-en-17203/332186388 (accessed on 25 July 2021). [CrossRef]

39. Koes, D.R.; Camacho, C.J. ZINCPharmer: Pharmacophore search of the ZINC database. Nucleic Acids Res. 2012, 40, W409-W414. [CrossRef] [PubMed]

40. Feigl, F.; Suter, H.A. Analytical Use of Sodium Rhodizonate. Ind. Eng. Chem. Anal. Ed. 1942, 14, 840-842. [CrossRef]

41. Jia, X.; Li, H.; Luo, J.; Lu, Q.; Peng, Y.; Shi, L.; Liu, L.; Du, S.; Zhang, G.; Chen, L. Rational Design of Core-Shell Molecularly Imprinted Polymer Based on Computational Simulation and Doehlert Experimental Optimization: Application to the Separation of Tanshinone IIA from Salvia Miltiorrhiza Bunge. Anal. Bioanal. Chem. 2012, 403, 2691-2703. [CrossRef] [PubMed]

42. Yan, M.; Ramström, O. Molecularly Imprinted Materials: Science and Technology; Yan, M., Ed.; CRC Press: Boca Raton, FL, USA, 2020.

43. Carro-Diaz, A.M.; Lorenzo-Ferreira, R.A. Molecularly imprinted polymers for sample preparation. In Handbook of Molecularly Imprinted Polymers; Alvarez-Lorenzo, C., Concheiro, A., Eds.; Smithers Rapra Technology: Shawbury, UK, 2013 ; pp. 87-127. 